\title{
El modelo de acción del Observatorio de Innovación Social, en el marco del Parque Científico de Innovación Social
}

\author{
Daniel Rocha-Jiménez ${ }^{\star}, H_{0}$ tencia Rueda-Lizarazo ${ }^{* *}$, Rosa Alexandra Chaparro-Guevara***
}

\footnotetext{
M.Sc. en Administración de Empresas. Director de Gestión del Conocimiento, Corporación Universitaria Minuto de Dios, Bogotá, Colombia. Correo electrónico: danielrochajimenez@gmail.com

** M.Sc. en Economía. Docente investigador, Universidad Militar Nueva Granada, Bogotá, Colombia. Correo electrónico: hruedal@gmail.com

*** Candidato a Doctor. Investigador senior, Observatorio de Innovación Social, Corporación Universitaria Minuto de Dios, sede Bogotá, Colombia. Correo electrónico: rchaparro@gmail.com
}

Recibido: 21 de enero del 2016 Aceptado: 31 de mayo del 2016

Cómo citar este artículo: Rocha-Jiménez, D., Rueda-Lizarazo, H., Chaparro-Guevara, R. A. (2016). El modelo de acción del Observatorio de Innovación Social en el marco del Parque Científico de Innovación Social. Cooperativismo \& Desarrollo, 24(109), xx-xx. doi: 10.16925/co.v24i109.1509

\section{Resumen}

Propósito: el artículo pretende explicar la acción del Observatorio de Innovación Social (ois) en búsqueda del foco estratégico del Parque Científico de Innovación Social (pcis), que está relacionada con la transformación de comunidades y rastrea la gestión del conocimiento y de los procesos, los métodos y las herramientas del ois. Temas: el pcis tiene un modelo de gestión particular, que se hace complejo por la combinación del término distante de "parque científico", derivado, por un lado, de la economía de mercado y, por el otro, del concepto de innovación social, aún en construcción, polivalente y polisémico, asociado con problemáticas y retos de la sociedad. Desarrollo: en este marco se encuentra el ois, unidad de gestión del pcis dedicada a la vigilancia del entorno en búsqueda de oportunidades y retos tanto en las comunidades como en los medios utilizados para atender dichos retos como el conocimiento y las tecnologías, el cual está operando de forma ajustada al modelo aquí mostrado desde el 2013 a la fecha. Conclusiones: el ois tiene tareas importantes en la observación de los procesos de transformación de comunidades, bien sea como resultados o bien impactos, así como los procesos previos asociados a la vigilancia del entorno, de observación de los saberes tradicionales, de oportunidades, retos y tecnologías.

Palabras clave: gestión del conocimiento, innovación social, observatorio de innovación social, transformación de comunidades. 


\title{
The action model of the Social Innovation Observatory of the Social Innovation Science Park
}

\begin{abstract}
Purpose: This article aims to explain the actions of the Social Innovation Observatory (ois by its Spanish initials) that are aligned with the strategic focus of the Social Innovation Science Park (pcis), which are related to transforming communities and tracking the management of knowledge and ois processes, methods, and tools. Topics: The pcis has a particular model of management, complex for its combination of the obscure term "science park" that is derived, on the one hand, from the market economy and, on the other, the polyvalent and polysemic concept of social innovation that is still under construction and is associated with the problems and challenges of society. Development: In this context we find the ois, a management unit of the pcis that is dedicated to monitoring the environment in search of opportunities and challenges in communities. It also searches for means to address these challenges such as knowledge and technologies. Its operations have closely adhered to the model shown here since 2013. Conclusions: The ois fulfills an important role in the observation of the processes of community transformation, both outcomes and impacts, as well as those processes related to environmental monitoring. It additionally observes traditional knowledge, opportunities, challenges, and technologies.
\end{abstract}

Keywords: knowledge management, social innovation, social innovation observatory, community transformation

\section{O modelo de ação do Observatório de Inovação Social, no âmbito do Parque Científico de Inovação Social}

\section{Resumo}

Propósito: este artigo pretende explicar a ação do Observatório de Inovação Social (ois) em busca do foco estratégico do Parque Científico de Inovação Social (pcis), que está relacionada com a transformação de comunidades e rastreia a gestão do conhecimento e dos processos, os métodos e as ferramentas do ois. Temas: o pcis tem um modelo de gestão particular, que se torna complexo pela combinação do termo distante de "parque científico", derivado, por um lado, da economia de mercado e, por outro, do conceito de inovação social, ainda em construção, polivalente e polissêmico, associado com problemáticas e desafios da sociedade. Desenvolvimento: nesse âmbito encontra-se o ois, unidade de gestão do pcis dedicada à vigilância do meio em busca de oportunidades e desafios tanto nas comunidades quanto nos meios utilizados para atender esses desafios como o conhecimento e as tecnologias, o qual está operando de forma ajustada ao modelo aqui mostrado desde 2013 até a data. Conclusões: o ois tem tarefas importantes na observação dos processos de transformação de comunidades, seja como resultados seja como impactos, bem como os processos prévios associados à vigilância do meio, de observação dos saberes tradicionais, de oportunidades, desafios e tecnologias.

Palavras-chave: gestão do conhecimento, inovação social, observatório de inovação social, transformação de comunidades. 


\section{Introducción}

\section{Parque Científico ${ }^{1}$ de Innovación Social}

El concepto de parque es un espacio de apertura, de libre circulación de actores, de flujo sin restricciones y de uso colectivo.

Parque científico es una denominación más formalizada que posee fronteras muy precisas al vincular una unidad de gestión con un entorno universitario y de investigación.

Para la Asociación Internacional de Parques Tecnológicos ${ }^{2}$ (iasp, por sus siglas en inglés), un parque científico es una organización gestionada por profesionales especializados, cuyo objetivo fundamental es incrementar la riqueza de su comunidad promoviendo la cultura de la innovación y la competitividad en las empresas e instituciones generadoras de saber, instaladas este, propósito que en esencia se vincula con los procesos de transferencia de conocimiento.

De esta manera, el concepto de parque científico, tal como se entiende desde la iasp, vincula procesos de generación de riqueza, crecimiento económico y desarrollo empresarial, por lo que, en una comprensión más intuitiva de los parques científicos, promueven el desarrollo económico, a partir de la creación de valor para la sociedad a través de nuevas empresas y productos; este concepto está completamente alineado con el de innovación y con el de la creación de valor económico.

El Parque Científico de Innovación Social (PCIS), unidad de gestión creada en el 2010 y gestionada por la Corporación Universitaria Minuto de Dios -uniminuto $^{3}$ - ha rescatado no solo una noción de valor económico, sino de valor social ${ }^{4}$. En su concepción,

\footnotetext{
1 Parque Científico de Innovación Social (PCIS): definimos el PCIs como una plataforma de relacionamiento, física y tecnológica que promueve y trabaja en la solución a problemáticas sociales, la integración de actores, la aplicación de métodos innovadores, la dinamización de modelos replicables, la visibilización de prácticas y experiencias en innovación social., la apropiación social del conocimiento y el fortalecimiento de capacidades de innovación social en las regiones.

2 http://www.iasp.ws/home

3 La Corporación Universitaria Minuto de Dios donde se en cuentra alojado el PCIs es una institución universitaria que atiende más de 110 mil estudiantes en Colombia.

$4 \quad$ "Valor social", en el contexto del PCIS, se entiende como un cambio positivo en las condiciones de vida de una comunidad, representado en condiciones de bienestar en aspectos tangibles (infraestructura, nivel de ingreso o acceso a servicios) o intangibles (convivencia en la diversidad, ciudadanía activa, solidaridad u otros)
}

el PCIS puede ser considerado como un agente de gestión en el ecosistema de innovación de la cuádruple hélice: universidad, empresa, Estado y sociedad. En este ecosistema existe un gran compromiso con la generación de valor económico a través de la creación de empresas y productos, también hay un interés en la creación de valor social mediante las mejoras en la calidad de vida de las comunidades.

Sin embargo, el PCIs como agente del ecosistema de innovación que se estructura a partir de la concepción de parque científico busca tener una mayor relevancia por encontrarse en un entorno universitario y de investigación, y no en uno de generación de empresas y de nuevos productos. Lo anterior lo convierte en un modelo con características particulares, enmarcado en la enorme complejidad que el concepto de innovación social -concepto polisémico, polivalente y en permanente construcción-imprime a cada uno de sus frentes de gestión (Lora y Rocha, 2015).

\section{La innovación social y el modelo del PCIS}

Un modelo es siempre una representación reduccionista, sesgada, simplista de la realidad, una aproximación defectuosa al objeto que pretende representar, llena de vacíos y limitaciones; no obstante, es potente en su simplicidad para el proceso de convertir el conocimiento tácito en explícito.

En el caso del PCIs, desde el 2010 se ha ido gestando un proceso constante de construcción de una definición estratégica, conceptual y operacional de este tipo particular de parque científico, proceso inmerso necesariamente en las ambigüedades propias de estos emprendimientos. Este proceso, lleno de aciertos, errores y contradicciones ha sido guiado por diversos enfoques, traslapados entre sí, asimétricos, que requieren ser formalizados.

La apuesta del modelo que orienta la gestión del PCIS es la "búsqueda e implementación de soluciones novedosas, eficientes, participativas y sustentables a los problemas que limitan el logro de mejores condiciones de vida en una comunidad" (PCIS, 2011). En las líneas anteriores se identifica que en el PCIs se crisolan dos conceptos, en principio distantes y contradictorios: por un lado, el de parque científico, asociado con el valor económico, el emprendimiento, la empresa, el innovador y el individuo, y, por el otro, la noción de innovación social, vinculada con la generación de valor social, el colectivo, el bienestar y los nuevos papeles de la sociedad. 
¿Cómo se resuelve en la práctica la conjunción entre estos dos escenarios aparentemente distantes y contradictorios? La apuesta del PCIS se ha dado a través de la búsqueda de una complementariedad entre procesos que incrementan la riqueza de la comunidad y los que las transforman; esto promueve la cultura de la innovación y la competitividad de la mano de una gestión encaminada a la transformación de las comunidades que se encuentran vinculadas a los procesos desarrollados por el PCis en sus diferentes ámbitos de acción, como se puede observar en la figura 1 .

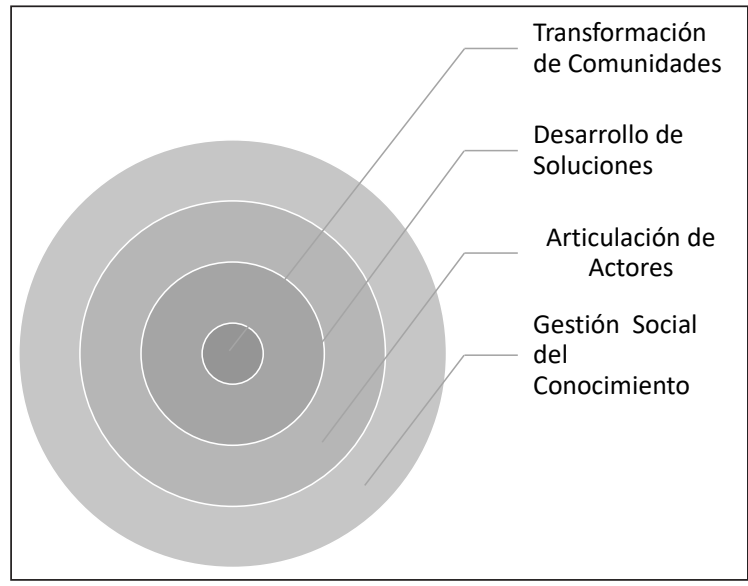

Figura 1. Modelo simplificado de gestión del PCis. Elaboración propia.

La conceptualización misma de la categoría de innovación social da cuenta de la necesidad de caracterizarla como un escenario potencial de transformaciones que, de alguna manera, busca solventar la concentración excesiva del modelo clásico de innovación en el desarrollo tecnológico y la generación de nuevos productos, identificando este campo, inclusive, como un espacio de gestión útil para resolver los fallos del Estado y del mercado, al abordar diversos desafíos como la pobreza, el envejecimiento de la población, la inclusión de grupos sociales más vulnerables, el cambio climático, etc. (Conejero, 2015; Morales, 2008).

Para la Organización, la Cooperación y el Desarrollo (ocde), la innovación social busca nuevas respuestas a los problemas sociales, mediante la identificación y provisión de nuevos servicios, procesos, competencias y formas de participación que mejoren la calidad de vida de las comunidades. Para la Comisión Europea, se trata de una apuesta por la experimentación social, asociada a métodos participativos y de colaboración, de codiseño y coconstrucción, además de ser alternativas adaptadas a las nuevas realidades sociales. Para Conejero (2015), la innovación social puede ser vista como el resultado de un aprendizaje colectivo en red, en el cual participan actores públicos, privados y del tercer sector, con el objetivo compartido de mejorar problemas sociales que pueden ser un factor decisivo en la cohesión social.

Para Morales (2008), es indispensable identificar los aspectos que diferencian la innovación social de la innovación económica y-tecnológica; la última es intensiva en capital financiero y tecnológico, y busca desarrollar productos que generen alta rentabilidad y ventajas para los competidores; por su parte, las innovaciones sociales son intensivas en capital intelectual (el cual está centrado en procesos de gestión y transferencia de conocimiento), tienen bajos costos y gran impacto. En el capital social-relacional, se transforma el modelo de gobernanza, pues esta llega a ser menos centralizada e involucra diferentes niveles de gestión entre lo local y lo global. Esto se hace a través, de nuevas y más eficientes interacciones entre el Estado, el sector privado y la ciudadanía territorial.

En la búsqueda de la definición estratégica, conceptual y operacional del pCis entran en juego muchos aspectos señalados en la literatura sobre innovación social. Esto se logra, bien sea por la identificación de nuevas respuestas, procesos, competencias y formas de participación para la solución de problemas sociales, bien desde la promoción de nuevas y más eficientes estrategias de gobernanza, ya sea a partir de la interacción con diversos actorelocales y globales, ya desde el aprendizaje colectivo y en red. Por último, todo esto se encuentra mediado por el aprovechamiento del capital intelectual científico y del conocimiento acumulado por las comunidades locales para formular y desarrollar alternativas a problemas sociales (gestión del conocimiento). El PCIs concibe su modelo de gestión como un escenario real de transformación de las comunidades (Lora y Rocha, 2015).

\section{Observatorios sociales}

Antes de comenzar con la descripción del Observatorio de Innovación Social (oIs) ${ }^{5}$ es importante, oIs reconocer algunos conceptos comunes que en el ámbito mundial se trabajan alrededor de observatorios, ya que por la diversidad de enfoques y procesos, cada uno desarrolla sus actividades de

El Observatorio de Innovación Social está operando de forma ajustada al modelo aquí mostrado desde el 2013 a la fecha. 
forma diferente. Esto es así tanto los observatorios sociales (Urdapilleta, 2006), cuyo objetivo es evaluar y hacer seguimiento a las problemáticas de orden social, como aquellos de carácter sectorial en los cuales se realiza una recopilación sistemática y permanente de datos, con el fin de procesarla y entregarla como información dotada de importancia y propósito para un público interesado en esta (iica, 2006).

Continuando con el rastreo de algunas definiciones, se identifica la de Ortuño (2006), quien reconoce a un observatorio como un punto desde el cual se observa un objeto o una situación: se puede entender como un proceso de monitoreo de tendencias observables y medibles. Según Angulo (2009), la misión de un observatorio es identificar la información, reconocer la importancia y presentarla de forma clara, es decir, que una característica tiene que ver con la recopilación de datos y su procesamiento, con el fin de entregar información pertinente en torno al tema de cada observatorio.

Se pueden encontrar en el mundo diferentes tipos de observatorios: por el tema que trabajan, por el sector en el que observan o por la cobertura que tengan. No obstante, ahí no termina la forma de clasificar los observatorios: otra forma es a partir de las funciones que realiza. Para Prieto (2003), los observatorios tienen dos propósitos: investigar la recopilación de los datos, la revisión y la descripción hasta llevar la información a diversos tipos de comunidades. Por lo anterior, es importante que un observatorio identifique su tema de interés y las metodologías que utilizará para desarrollar sus mediciones. El oIs se reconoce como un espacio que permite desarrollar pensamiento, que recopila y analiza datos bien sea de fuentes primarias o secundarias, que tiene la capacidad de encontrarse con la complejidad de diferentes realidades y entornos, con actitud abierta al conocimiento y con capacidades de interrelacionamiento de diferentes actores que trabajen el tema de la innovación social.

Es decir que uno de los elementos que caracteriza a la sociedad del conocimiento tiene que ver con la capacidad de articular las tic y la sociedad, razón por la cual desde el trabajo que realiza el ois orsse desarrollan espacios, a través de la plataforma de conocimiento que tiene el PCIs, un espacio en el cual los profesionales del Parque y la comunidad interesada en la innovación social, interactúan en la construcción colaborativa de metodologías y documentos de reflexión alrededor del quehacer del observatorio.

\section{El oIs, al interior del PCIS}

El ors, en el modelo de gestión del conocimiento del PCIS, aparece como un macro proceso asociado con la creación de conocimiento. Se convierte la observación en la forma básica de investigación, entendida como el primer paso de todo ejercicio investigativo a partir de la captura, recopilación y análisis de la información.

El ois desarrolla acciones de creación de conocimiento a través de métodos cuantitativos y cualitativos de investigación, basado en fuentes primarias y secundarias, los estudios de caso y el desarrollo de estudios sobre impactos y sobre resultados, lo que genera múltiples documentos y actividades que facilitan la apropiación social del conocimiento, así como su divulgación en el entorno académico, empresarial y estatal. Ahora bien, es importante describir de una manera breve el modelo de gestión del PCIs y su relación con oIs, que se muestra en la figura 2.

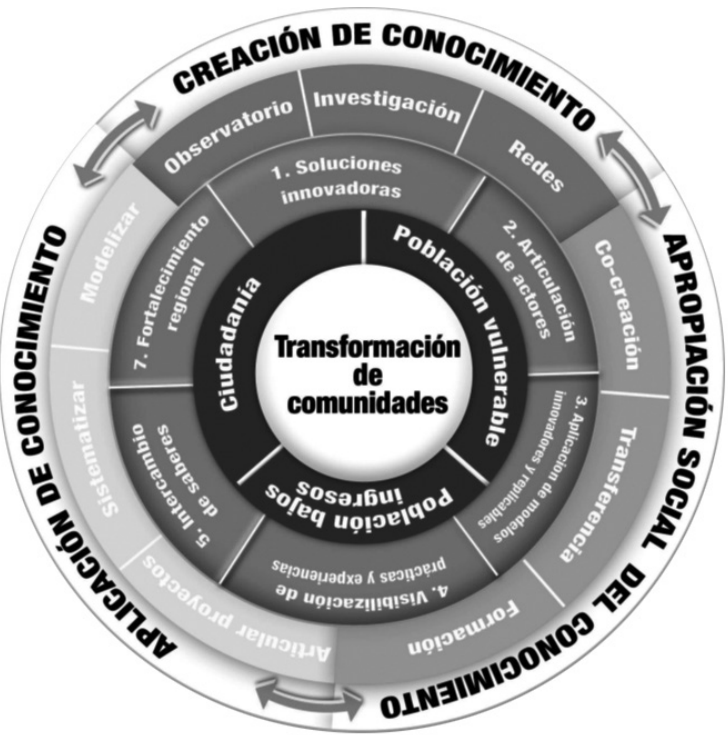

Figura 2. Modelo de Gestión del Conocimiento del PCIs. Elaboración propia.

El modelo de gestión del oIs está enmarcado en dos rutas orientadoras: por un lado, la ruta de los interesados y los beneficiarios, y, por la otra, la ruta de la gestión del conocimiento, centrada en los procesos del observatorio y que busca facilitar los procesos asociados al foco estratégico del PCIs, a saber, la transformación de comunidades. En otras palabras, dado que la transformación de comunidades se puede dar a partir del cambio de 
las condiciones de vida de cualquier individuo o grupo de individuos, este cambio no solo implica elementos físicos, sino condiciones personales de cada sujeto. El ors tiene un trabajo importante para el desarrollo de estos objetivos, como, la identificación de oportunidades, nuevas tecnologías y saberes de las comunidades.
También es relevante para el oIs la observación de los resultados e impactos buscados y logrados por el PCIS y terceros, con el objetivo de lograr transformación en las comunidades. Con lo anterior, se devela una actividad intrínseca y básica del ois, que se encuentra asociada a la construcción de metodologías y a las herramientas para lograr tal cometido, como se puede observar en la figura 3.

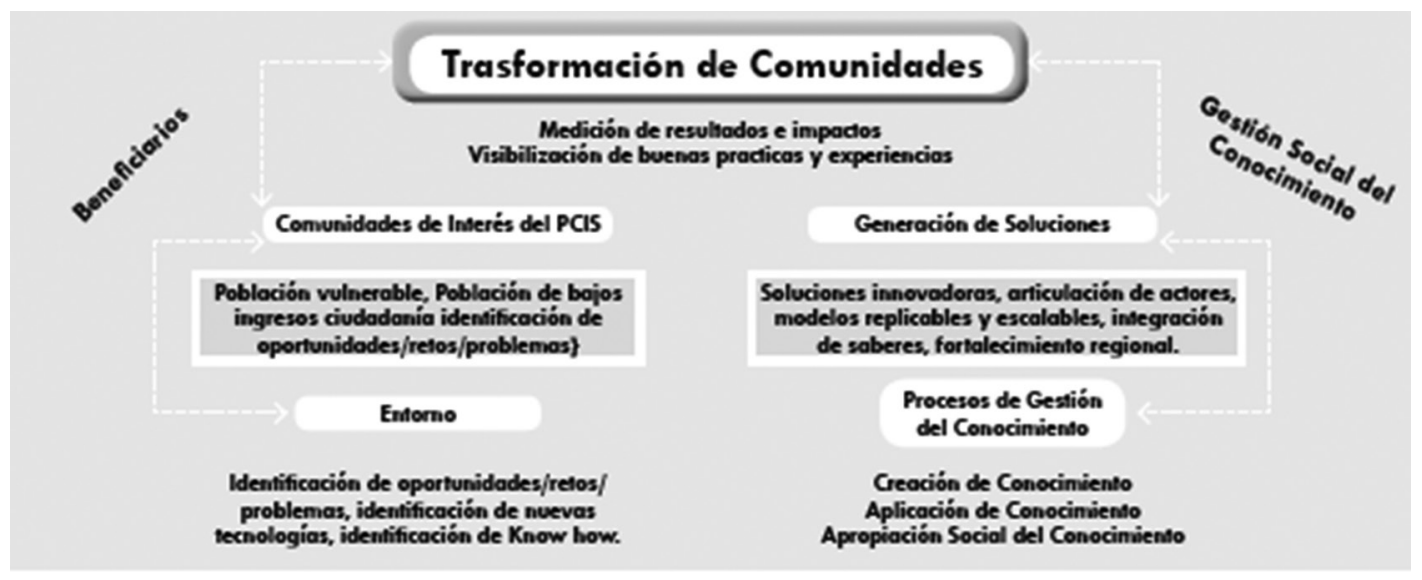

Figura 3. Marco de actuación del ois al interior del pcis. Elaboración propia.

El ois es un ente dinámico en el que interactúan diferentes procesos ${ }^{6}$ que se pueden ver en la figura $4 \mathrm{y}$ entre los cuales se encuentran:

- La cocreación: es el proceso mediante el cual se participa con los diferentes actores. Como lo manifiestan Kotler y Kotler (2014), la organización no se puede limitar a observar el comportamiento de los actores, sino que debe invitar a que participen en la creación y al desarrollo de ideas y productos. La anterior es una mirada desde el consumo, pero muy relacionada al concepto de cocreación: se trata de generar las herramientas para que se puedan expresar las ideas y llegar a un ejercicio de prototipado participativo. Para la consultora McKinsey la cocreación tiene que ver

\footnotetext{
6 Como ejemplos de los diferentes procesos y sus productos podemos mencionar:

a. Talleres de cocreación para desarrollar la prospectiva del Conservatorio del Tolima;

b. Cartografía social de Cundinamarca, realizada con el propósito de identificar, documentar y mapear las problemáticas sociales.

c. Sistematización de los 13 años del Premio Cívico por una Ciudad Mejor.

d. Documentación del premio Fundación el Nogal.

e. Medición del impacto de los egresados de uniminuto.
}

con la innovación abierta en la cual existe participación de expertos, organizaciones e individuos con el fin de lograr un beneficio común (Barbá, 2011).

- Vigilancia del entorno: es la actividad que realizan las organizaciones que tienen como finalidad determinar los cambios en torno al desarrollo humano, social y tecnológico (Riba, 2002).

- Generación de metodologías e instrumentos: en esta fase de trabajo, el ors identifica las metodologías de trabajo con las cuales se acerca al territorio y utiliza instrumentos que le permitan levantar información y desarrollar actividades con las comunidades.

- Análisis de fuentes primarias y secundarias: se entiende como fuente primaria aquella que recopila el ois a través de las propias caracterizaciones en territorio o de forma directa por medio de los proyectos o talleres que realizan otras unidades del PCIs. Las fuentes secundarias son los estudios o datos que se analizan o se toman de otras entidades como referente de temáticas propias del Observatorio.

- Documentación y modelización: una vez concluida la etapa de levantamiento de información de fuentes primarias o secundarias, se procede a 
documentar las actividades y luego a modelarlas; algunos casos que se han desarrollado en el observatorio son: mediciones de impacto, sistematización de experiencias, análisis prospectivo, cartografías sociales, entre otras.

En relación con la ruta de los interesados, la finalidad del PCIs, que se alinea con la del ois, es la transformación de comunidades, que se entiende como el resultado de la creación de "valor social", esto es, un cambio positivo en las condiciones de vida de una comunidad, representado en condiciones de bienestar, aspectos tangibles (infraestructura, nivel de ingreso o acceso a servicios) o intangibles (convivencia en la diversidad, ciudadanía activa, solidaridad u otros; Rocha, 2015).

Como consecuencia del anterior trabajo, se presentan soluciones innovadoras que propenden la articulación de actores en busca de modelos replicables y de un fortalecimiento regional que aporte a la apropiación social del conocimiento de los actores participantes.

El ois busca generar valor a las soluciones generadas por el PCIs, a partir de un conjunto de procesos y productos y a través de la gestión social del conocimiento. Este valor está asociado con la identificación de oportunidades, retos y problemáticas, la visibilización de buenas prácticas y la sistematización de experiencias.

De esta manera, para mostrar el marco de trabajo del ois, brevemente se enlistan los productos del observatorio.

- Talleres: son espacios en los cuales se une la teoría y la práctica con el fin de contextualizar algunas formas de interacción y encontrar posibles soluciones. Generalmente los talleres tienen un facilitador que orienta los procesos, pero no solamente de forma unidireccional, sino con el objetivo de recopilar información para construir conocimiento, a partir de las situaciones analizadas (Universidad de Antioquia, s.f.).

- Mapeo y caracterización: es un proceso mediante el cual se describen e identifican acontecimientos o fenómenos que se investigan (Sánchez-Upegüi, 2010). La caracterización es una conceptualización principalmente cualitativa, que incluye elementos cuantitativos para la identificación y organización de información sobre realidades en un lugar y momento determinado. Se puede constituir como una de las primeras fases de los métodos de sistematización de datos y experiencias.

La caracterización hace parte de uno de los espacios de generación del reconocimiento de las realidades sociales y del territorio, en el cual se van a desarrollar o se vienen desarrollando innovaciones sociales, por lo cual, esta información se conforma en un insumo para establecer la línea de base sobre la cual se miden los impactos generados en las comunidades y sus territorios.

- Metodologías: investigación acción participativa. Se comprende como una vivencia que articula actitudes y valores en un método de trabajo orientado a la praxis (Fals-Borda, 1999). Se ve a la investigación acción participativa como una filosofía de vida; los participantes son seres sentipensantes, es decir, aquel que es capaz de combinar la razón, el amor, el cuerpo y el corazón. Este concepto se inspira en la forma de vivir de los pescadores de la costa caribe colombiana. En otras palabras lo que la investigación acción participativa desarrolla es la capacidad de generar conocimiento, por un lado, a partir del reconocimiento y conocimiento de una realidad, y, por el otro, de procesos de reflexión y de construcción en colectivo, buscando la transformación social (Fals-Borda, 2009).

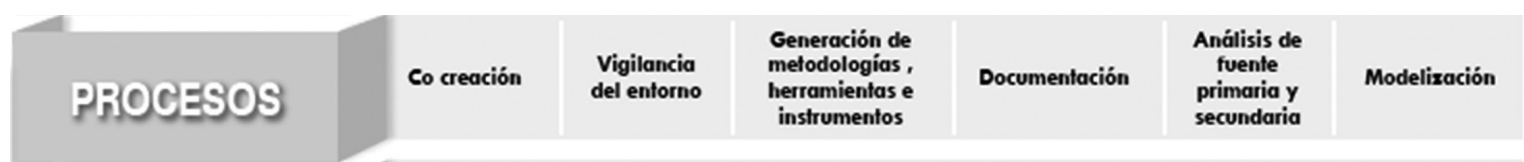

Figura 4. Procesos del ois. Elaboración propia.
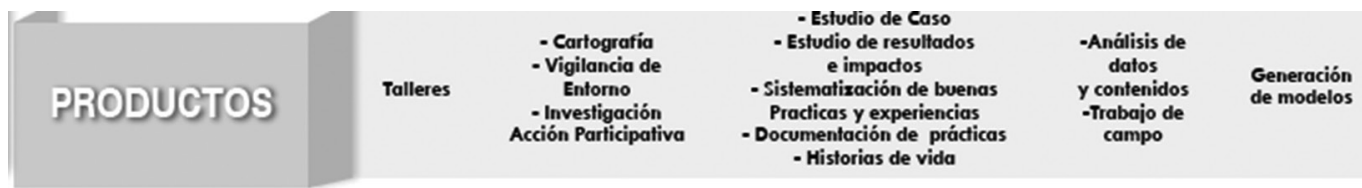

Figura 5. Productos del ois. Elaboración propia. 


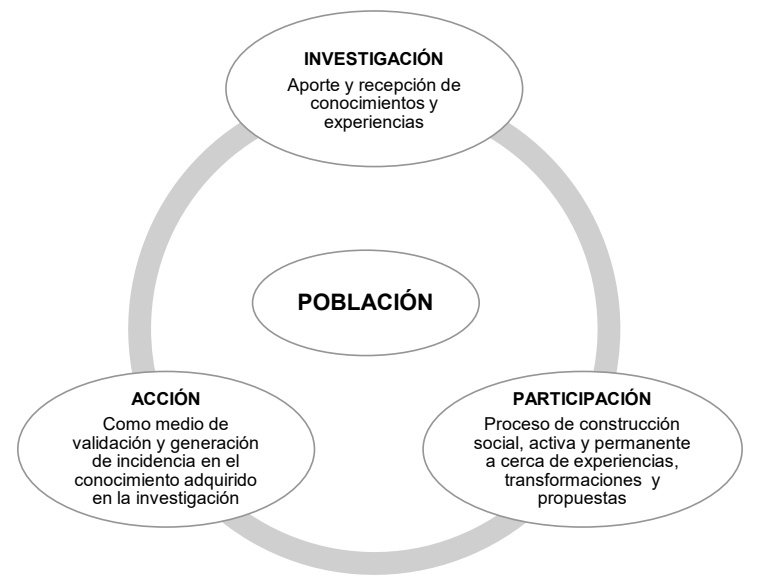

Figura 6. Proceso metodológico de la IAP.Tomado del Observatorio PCIS como resultado de una reflexión en el 2014.

Estudios de caso: son un método que se usa especialmente para analizar situaciones y entenderlas por medio de ejercicios de descripción. También se usa para estudiar los temas con una mirada multidisciplinar desde diferentes perspectivas, volviéndolos interesantes porque se obtienen miradas más profundas y se consigue un conocimiento amplio sobre cada fenómeno que se está estudiando. La finalidad de un estudio de caso es reconocer cómo funcionan todos los elementos que hacen parte del caso con el fin de generar hipótesis (Bisquerra, 2009).

Estudios de resultados e impacto: son aquellos en los que hay una medición de elementos a corto y largo plazo. En la evaluación de impacto, se miden los cambios que se suscitaron una vez se implementa el programa: son estudios más rigurosos en los cuales hay grupos de control y de comparación.

Sistematización de buenas prácticas y experiencias: la sistematización de experiencias en América Latina toma importancia en la década de los ochenta gracias al interés por documentar los procesos, aprendizajes y participación de las comunidades. La sistematización se entiende como una:

Interpretación crítica de una o varias experiencias que, a partir de su ordenamiento y reconstrucción, descubre o explicita la lógica del proceso vivido, los factores que han intervenido en dicho proceso, cómo se han interrelacionado entre sí y por qué lo han hecho de ese modo (Francke y Morgan, 1995).

Se entiende entonces por "sistematización" una metodología que permite la recolección y generación de conocimiento permanente, a partir de las experiencias que surgen de una realidad.

- Historias de vida y memoria histórica: ayuda a registrar de forma sistemática los procesos del individuo y de los grupos. Aquí es importante la reconstrucción del lenguaje ya que a través de este se expresan los pensamientos y deseos de los sujetos (Puyana y Barreto, 2010).

- Cartografía social: es una metodología que permite aproximarse a las realidades a partir de la construcción de un conocimiento integral en el que participan los habitantes, quienes expresan sus elementos vivenciales desde la cotidianidad. La cartografía hace uso de la construcción del mapa en el que la comunidad dibuja su realidad. Generalmente se construye desde lo más sencillo hasta la construcción de estructuras de realidades (sena, 2009).

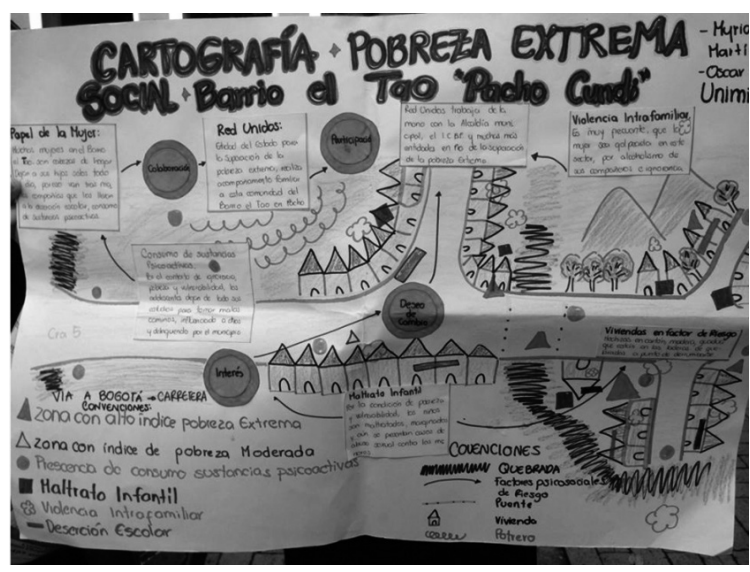

Figura 7. Proceso metodológico de la cartografía social. Tomada del Observatorio de Innovación Social, sede Cundinamarca (Colombia), como resultado de una práctica en responsabilidad social con estudiantes, en el segundo semestre del 2014.

Con el anterior ejemplo se puede mostrar que la cartografía social es importante porque hay una construcción colectiva de los ambientes reales de las comunidades. Para esto, se debe tener en cuenta el proceso metodológico como se aprecia en la figura 8.

- Generación de modelos: con base en la sistematización de experiencia, las buenas prácticas y la vigilancia del entorno, el ors construye modelos de trabajo que permiten el posterior diseño de proyectos de interacción con comunidades. Estos modelos son formas simplificadas de comprender la realidad y de formalizar el conocimiento sobre los fenómenos estudiados. 

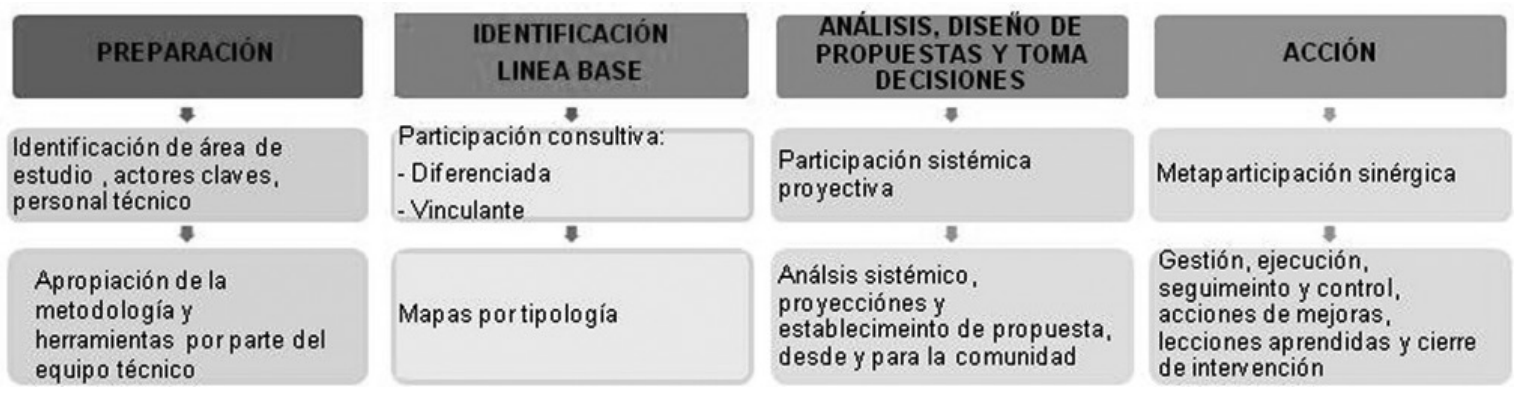

Figura 8. Procesos del observatorio de innovacion social. Elaboración propia.

\section{Conclusiones}

El PCIs posee un modelo de gestión particularmente complejo asociado a la combinación de un concepto bastante nuevo y en construcción como el de innovación social. El de parque científico está asociado más a la creación de valor económico.

Los ois se pueden clasificar en diversas tipologías de acuerdo a los enfoques, temas tratados y a la finalidad buscada.

El modelo del ors busca cumplir el modelo del PCIs, que pretende la transformación de comunidades a través de la mejora de la calidad de vida de las personas.

En ese sentido, el ors tiene tareas importantes en la observación de los procesos de transformación de comunidades, bien como resultados, bien como impactos, así como los procesos previos asociados a la vigilancia del entorno, de observación de los saberes tradicionales, de oportunidades, retos y tecnologías.

$\mathrm{El}$ ors tiene un conjunto de procesos internos que genera productos y documentos, y que pretende ser de utilidad para las comunidades y para el PCIs.

El modelo del ors está regido por el modelo de gestión del conocimiento del PCIs, la finalidad asociada a la transformación de comunidades, y un conjunto de procesos y productos vinculados a su función básica de creación de conocimiento (observar) y generar productos de información de diversos tipos.

\section{Referencias}

Angulo, N. (2009). ¿Qué son los observatorios y cuáles son sus funciones? Innovación Educativa, 9(47), 5-17.

Barbá, E. (2011). Innovación: 100 consejos para inspirarla y gestionarla. Barcelona: Libros de Cabecera.
Bisquerra, R. (2009). Metodología de la investigación educativa. Madrid: La Muralla.

Fals-Borda, O. (1999). Orígenes universales y retos actuales de la investigación-acción participativa. Análisis Político, 38.

Fals-Borda, O. (2009). Una sociología sentipensante para América Latina. Bogotá: Siglo del Hombre.

Francke, M. y Morgan, M. D. (1995). La Sistematización: Apuesta por la Generación a partir de las experiencias de promoción. Materiales Didácticos, 1-34.

iica. (2006). Observatorio iica. Paraguay: iica. Recuperado de: https://books.google.com.co/ books?id=5tLPY6YHhWcC\&pg=PA23\&dq=iica + observatorios + paraguay $\&$ hl=es $-419 \&$ sa $=\mathrm{X} \& v e-$ d=0ahUKEwj2gorBo6PPAhWCOiYKHT7rCmkQ6AEILzAD\#v=onepage\&q\&f=false

Kotler, P. y Kotler, M. (2014). 8 maneras de crecer. Madrid: lid.

Lora, P. y Rocha, D. (2016). Promoción de la innovación social a través de la utilización de metodologías participativas en la gestión del conocimiento. Equidad \& Desarrollo, 25, 159-178. doi: http://dx.doi. org/10.19052/ed.3513

Ortuño, C. (2006). Observatorios: Una mirada exploratoria, primer taller el observatorio como herramienta para la gestión de la información y del conocimiento. Caracas: sela.

Puyana, Y. y Barreto, J. (2010). La historia de vida: recurso en la investigación cualitativa Reflexiones Metodológicas. Maguaré.185-196. Recuperado de http://www. bdigital.unal.edu.co/18451/2/14265-48104-1-PB.pdf

Rocha, D. (2015). Infografía del Modelo de Gestión del Conocimiento del Parque Científico de Innovación Social. 
SENA. (2009). Formación en gestión ambiental y cadenas productivas sostenibles: herramienta de trabajo en cartografía social. Recuperado de http://tropenbos.sena. edu.co/DOCUMENTOS/HERRAMIENTAS\%20METODOLOGICAS/5\%20Guia_cartografia_social.pdf

Universidad de Antioquia. (s. f.). Conceptos Básicos de qué es un taller participativo, cómo organizarlo, cómo dirigirlo, cómo evaluarlo. Medellín: Centro de estudios de opinión. Recuperado de http://bibliotecadigital.udea. edu.co/bitstream/10495/2536/1/CentroEstudiosOpinion_conceptostallerparticipativo.pdf

Urdapilleta, A. M. (2006). Observatorio de violencia social y de género en Torreón. Recuperado de: www.lag.uia. $\mathrm{mx} /$ buenaval/buenaval2/Observatorio_de_violencia. pdf 